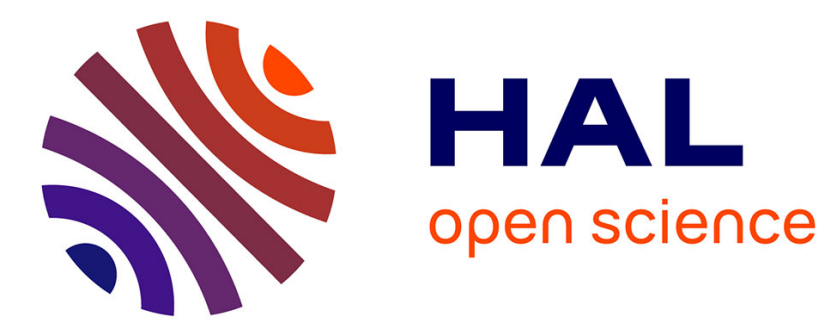

\title{
Some tractable instances of interval data minmax regret problems: bounded distance from triviality (short version)
}

\author{
Bruno Escoffier, Jérôme Monnot, Olivier Spanjaard
}

\section{To cite this version:}

Bruno Escoffier, Jérôme Monnot, Olivier Spanjaard. Some tractable instances of interval data minmax regret problems: bounded distance from triviality (short version). 34th International Conference on Current Trends in Theory and Practice of Computer Science, Jan 2008, Nový Smokovec, Slovakia. pp.280-291, 10.1007/978-3-540-77566-9_24 . hal-01302947

\section{HAL Id: hal-01302947 \\ https://hal.science/hal-01302947}

Submitted on 10 Jul 2017

HAL is a multi-disciplinary open access archive for the deposit and dissemination of scientific research documents, whether they are published or not. The documents may come from teaching and research institutions in France or abroad, or from public or private research centers.
L'archive ouverte pluridisciplinaire HAL, est destinée au dépôt et à la diffusion de documents scientifiques de niveau recherche, publiés ou non, émanant des établissements d'enseignement et de recherche français ou étrangers, des laboratoires publics ou privés. 


\title{
Some tractable instances of interval data minmax regret problems: bounded distance from triviality
}

\author{
Bruno Escoffier*, Jérôme Monnot*, and Olivier Spanjaard ${ }^{\dagger}$
}

\begin{abstract}
This paper focuses on tractable instances of interval data minmax regret graph problems. More precisely, we provide polynomial and pseudopolynomial algorithms for sets of particular instances of the interval data minmax regret versions of the shortest path, minimum spanning tree and weighted (bipartite) perfect matching problems. These sets are defined using a parameter that measures the distance from well known solvable instances. Tractable cases occur when the parameter is bounded by a constant. Two kinds of parameters are investigated, measuring either the distance from special weight structures or the distance from special graph structures.
\end{abstract}

Keyword Robust optimization; Interval data; Shortest path; Spanning tree; Bipartite perfect matching

\section{Introduction}

In recent years there has been a growing interest in robust optimization problems [15]. Studies in this field concern problems where some parameters are ill-known due to uncertainty or imprecision. Usually, in valued graph optimization problems, the ill-known parameters are the valuations. In such a case, a set of scenarios is defined, with one scenario for each possible assignment of valuations to the graph. Two approaches can be distinguished according to the way the set of scenarios is defined: the interval model where each valuation is an interval and the set of scenarios is defined implicitly as the cartesian product of all the intervals; the discrete scenario model where each valuation is a vector, every component of which is a particular scenario. Intuitively, a robust solution is a solution that remains suitable whatever scenario finally occurs. Several criteria have been proposed to formalize this: the minmax criterion consists of evaluating a solution on the basis of its worst value over all scenarios, and the minmax regret criterion consists of evaluating a solution on the basis of its maximal deviation from the optimal value

${ }^{*}$ LAMSADE-CNRS, Université Paris Dauphine, Place du $\mathrm{M}^{a l}$ de Lattre de Tassigny, F-75775 Paris Cedex 16, France, \{escoffier,monnot\}@lamsade.dauphine.fr

${ }^{\dagger}$ LIP6, Université Pierre et Marie Curie, 4 Place Jussieu, F-75252 Paris Cedex 05, France, olivier.spanjaard@lip6.fr 
over all scenarios. We will mainly focus here on the robust shortest path problem (RSP for short), the robust minimum spanning tree problem (RST for short) and the robust minimum weighted (bipartite) perfect matching problem $(\mathrm{R}(\mathrm{B}) \mathrm{PM}$ for short), with the minmax regret criterion in the interval model.

Formally, an interval data minmax regret network optimization problem can be defined as follows. Let $G=(V, E)$ be a given directed or undirected graph with $n$ vertices and $m$ edges. A feasible solution is a subset $\pi \subseteq E$ satisfying a given property $\Pi$ (for example, being a path, a tree or a matching). Each edge $e \in E$ is valued by an interval $I_{e}=\left[l_{e} ; u_{e}\right]$ of possible weights. The set of scenarios is the cartesian product $\mathcal{S}=\prod_{e \in E} I_{e}$. In other words, a scenario $s \in \mathcal{S}$ consists in assigning a weight $w_{s}(e) \in I_{e}$ for every $e \in E$. For any feasible solution $\pi$ and any scenario $s \in \mathcal{S}$ of an instance $\mathcal{I}=\left(G, I_{E}\right)$ where $I_{E}=\left\{I_{e}: e \in E\right\}$, the value of $\pi$ under scenario $s$ is $w_{s}(\pi)=\sum_{e \in \pi} w_{s}(e)$ and its regret under scenario $s$ is $R_{s}(\pi)=\left|w_{s}(\pi)-\operatorname{opt}(s)\right|$, where $\operatorname{opt}(s)$ is the value of an optimal solution for the standard instance valued by $w_{s}$ (rigorously, we should write $R_{s}(\mathcal{I}, \pi)$ but we omit to indicate $\mathcal{I}$ when no confusion is possible). The max regret of solution $\pi$ is defined by $R(\pi)=\max _{s \in \mathcal{S}} R_{s}(\pi)$. The aim of a minmax regret optimization problem is, given an instance $\mathcal{I}=\left(G, I_{E}\right)$, to find a feasible solution $\pi^{*}$ minimizing $R\left(\pi^{*}\right)$. Note that, for a minimization problem, $R(\pi)=R_{s(\pi)}(\pi)$, where $s(\pi)$, called worst case scenario for $\pi$, is defined by $w_{s(\pi)}(e)=u_{e}$ if $e \in \pi$ and $w_{s(\pi)}(e)=l_{e}$ otherwise $[3]$.

In this paper, we consider tractable instances of RSP and RST, that have been proved strongly NP-hard [4] in the general case, as well as tractable instances of RBPM, the restriction of which to complete bipartite graphs (known as the interval data minmax regret assignment problem) has been proved NP-hard [12]. For this purpose, as suggested by Guo et al. [10], we introduce parameters that measure the distance from well known solvable instances. For example, if all the intervals of an instance reduce to a single point-degenerate intervals-, then the robust optimization problem reduces to a standard optimization problem, and is therefore polynomially solvable provided that the standard version is polynomial. One can define the distance from this easy case as the number $k$ of non degenerate intervals. If this distance $k$ is bounded by a constant, then the robust optimization problem is polynomially solvable by a brute force algorithm [4]. In this work, we focus on two kinds of parameters: the ones that measure the distance from special valuation structures (instances the minmax regret of which is zero, instances with linearly ordered valuations), and the ones that measure the distance from special graph structures (series-parallel graphs, trees). The paper is organized as follows. The first two sections deal with the first kind of parameters: we show that RSP and RBPM are polynomially solvable when the minmax regret is bounded by a constant $k$ (Section 2), as well as RST when the number of intersecting intervals in the instance is bounded by a constant $k$ (Section 3 ). More precisely, following parameterized complexity terminology [8], the first two problems are in XP (problems solvable in $O\left(n^{f(k)}\right)$ for some function $f$ ) while the third one is in FPT (problems solvable in $O\left(f(k) n^{c}\right)$ for some constant $\left.c\right)$. The next sections deal with the second 
kind of parameters: we show that RSP is pseudopolynomial for graphs which are close to be series-parallel (Section 4), and that RSP and RBPM are pseudopolynomial for graphs with bounded treewidth and bounded degree (Section 5). Due to lack of space, some proofs are omitted and can be found in [9].

\section{Upper bounded minmax regret}

In this section, we investigate the hardness of solving an interval data minmax regret graph optimization problem when there exists a solution with bounded maximal regret. Note that studying instances where the optimum value is upper bounded is a classical way to understand the intrinsic difficulty of a combinatorial optimization problem (problems which become polynomially solvable in this case are called simple, see Paz and Moran [16]). Here, we first show that we can easily determine if there is a solution of maximal regret 0, i.e. a solution which is optimal under every possible scenario. Next, we show that for RSP and RBPM, we can extend this result to polynomially determine if there exists a solution of maximal regret at most $k$.

First, let us prove that the problem of the existence of a solution of maximal regret 0 can be easily solved for any interval data minmax regret graph optimization problem $\Pi$. We use a nice generic 2-approximation algorithm proposed by Kasperski and Zielinski [13]. For any instance $\mathcal{I}$ this algorithm outputs a solution $\pi$ such that $R(\pi) \leq 2 R\left(\pi^{*}\right)$ (where $R\left(\pi^{*}\right)$ is the minmax regret of $\mathcal{I}$ ). If $R\left(\pi^{*}\right)=0$, then $R(\pi)=0$, else since $R(\pi) \geq R\left(\pi^{*}\right)$, we have $R(\pi)>0$. The expected result follows ( $\Pi$ being assumed to be polynomial). Now, by a reduction to the regret 0 case, we prove the following:

Proposition 1 For $R S P$, the problem of determining if the minmax regret is at most $k$ can be solved in time $O\left(n^{2} m^{k}\right)$.

Proof. Let $\mathcal{I}=\left(G, I_{E}\right)$ be an instance of RSP and denote by $r$ its optimum regret. Let us remark that if there exists a degenerate interval $I_{e}=\{0\}$ in $\mathcal{I}$ with $e=\left(v_{1}, v_{2}\right)$, then one can merge nodes $v_{1}$ and $v_{2}$ and get an equivalent instance (possibly with multiedges). In particular, we can assume that $u_{e}>0$ for any $e$. We construct $m$ instances $\mathcal{I}_{1}, \ldots, \mathcal{I}_{m}$ of RSP as follows: $\mathcal{I}_{i}$ is the same instance as $\mathcal{I}$ up to the interval $\left[l_{i}, u_{i}\right]$ associated in $\mathcal{I}$ to $e_{i}$ which is transformed into $\left[\max \left\{l_{i}-1 ; 0\right\}, u_{i}-1\right]$. We claim that:

(i) $r_{i}^{*} \geq r-1$ where $r_{i}^{*}$ denotes the optimum regret of $\mathcal{I}_{i}$;

(ii) if $r_{i}^{*}=r-1$ then any optimum solution for $\mathcal{I}_{i}$ is optimum for $\mathcal{I}$;

(iii) there exists at least one $i$ such that $r_{i}^{*}=r-1$ (if $r>0$ ).

If the claims are true, then by applying $k$ times these procedures, $\mathcal{I}$ has an optimum regret at most $k$ if and only if (at least) one of the final instances has 
optimum regret 0 (if at some point, we find an interval reduced to $\{0\}$, we can merge the corresponding nodes). We get $m^{k}$ instances; the generic 2-approximation algorithm is in $O\left(n^{2}\right)$ for RSP, and the complexity follows. Claims $(i)$ and $(i i)$ hold since the regret of any path $\pi$ satisfies $R_{i}(\pi) \geq R(\pi)-1$ (under any scenario, the value of any path has decreased by at most 1$)$. For Claim (iii), consider an optimum solution $\pi^{*}=\left(\left(v_{0}, v_{1}\right), \cdots,\left(v_{p-1}, v_{p}\right)\right)$ (where $v_{0}=s$ and $\left.v_{p}=t\right)$ of $\mathcal{I}$, and its worst case scenario $s\left(\pi^{*}\right)$ in $\mathcal{I}$. We prove that there exists at least one edge $e_{i} \in \pi^{*}$ such that no shortest path in $s\left(\pi^{*}\right)$ contains this edge. Note that if this is true, then consider instance $\mathcal{I}_{i}$ : in $s\left(\pi^{*}\right)$, the value of the shortest path is the same in $\mathcal{I}$ and in $\mathcal{I}_{i}$, hence the regret of $\pi^{*}$ decreased by 1 , and Claim (iii) is true. Then, assume that for any $i$, there exists a shortest path $\pi^{i}$ (in $s\left(\pi^{*}\right)$ ) which contains $\left(v_{i-1}, v_{i}\right)$. Let $w_{1}^{i}$ be the value (in $\left.s\left(\pi^{*}\right)\right)$ of this path between $s$ and $v_{i-1}$ and $w_{2}^{i}$ its value between $v_{i}$ and $t$ (hence $\left.w_{1}^{1}=w_{2}^{p}=0\right)$. Since $\pi^{*}$ has regret $r$, we get $\left(s\left(\pi^{*}\right)\right.$ is omitted for readability) that $w\left(\pi^{i}\right)=w_{1}^{i}+w_{2}^{i}+u_{\left(v_{i-1}, v_{i}\right)}=w\left(\pi^{*}\right)-r$. Summing up we obtain:

$$
\sum_{i=1}^{p}\left(w_{1}^{i}+w_{2}^{i}\right)=p w\left(\pi^{*}\right)-p r-\sum_{i=1}^{p} u_{\left(v_{i-1}, v_{i}\right)}=(p-1) w\left(\pi^{*}\right)-p r
$$

But remark that for each $i \in\{2, \cdots, p\}$ we can build a path of value $w_{1}^{i}+w_{2}^{i-1}$ (composed of the initial part of $\pi^{i}$ from $s$ to $v_{i-1}$ and the final part of $\pi^{i-1}$ from $v_{i-1}$ to $\left.t\right)$. Then, since each of these paths has value at least $w\left(\pi^{*}\right)-r$ :

$$
\sum_{i=2}^{p}\left(w_{1}^{i}+w_{2}^{i-1}\right) \geq(p-1)\left(w\left(\pi^{*}\right)-r\right)=(p-1) w\left(\pi^{*}\right)-p r+r
$$

But since $w_{1}^{1}=w_{2}^{p}=0$, Equations (1) and (2) are incompatible for $r>0$.

The central property, leading to Claim (iii), is that, in an optimum solution $\pi^{*}$ for which $R\left(\pi^{*}\right)>0$, there exists at least one edge that does not belong to any optimum solution in $s\left(\pi^{*}\right)$. Actually, one can show that this property is also true for the interval data minmax regret perfect matching problem in bipartite graphs. For any instance $\mathcal{I}=\left(G, I_{E}\right)$ of $\mathrm{R}(\mathrm{B}) \mathrm{PM}$, we assume that $G$ has a perfect matching (in particular, the number $n$ of vertices of $G$ is even).

Proposition 2 For $R B P M$, the problem of determining if the minmax regret is at most $k$ can be solved in time $O\left(n^{2} m^{k}\right)$.

Proof. The proof is almost identical to the one of Proposition 1. Let $\mathcal{I}=\left(G, I_{E}\right)$ be an instance of RBPM where $G=(V, E)$ is a bipartite graph which admits a perfect matching and denote by $r$ its optimum regret. W.l.o.g., assume that $l_{e} \geq k$ for any $e$. Actually, by adding any constant $c>0$ to each interval $I_{e}$, we obtain an equivalent instance since all the perfect matchings have the same size. As previously, we build $m$ instances $\mathcal{I}_{1}, \ldots, \mathcal{I}_{m}$ of RBPM where $\mathcal{I}_{i}$ is the same instance as $\mathcal{I}$ up to the interval $\left[l_{i}, u_{i}\right]$ associated in $\mathcal{I}$ to $e_{i}$ which is transformed to $\left[l_{i}-1, u_{i}-1\right]$. 
Using the same notation as in Proposition 1, we claim that: $(i) r_{i}^{*}=R\left(\mathcal{I}_{i}\right) \geq r-1$; (ii) if $r_{i}^{*}=r-1$ then any optimum solution for $\mathcal{I}_{i}$ is optimum for $\mathcal{I}$; (iii) there exists at least one $i$ such that $r_{i}^{*}=r-1$ (if $r>0$ ).

The proof of Claims $(i)$ and $(i i)$ is identical to the proof of Proposition 1. So, we only prove Claim $(i i i)$. Consider an optimum solution $\pi^{*}=\left\{e_{1}, \cdots, e_{\frac{n}{2}}\right\}$ of $\mathcal{I}$, and its worst case scenario $s\left(\pi^{*}\right)$ in $\mathcal{I}$. As previously, we prove that there exists at least one edge $e_{i} \in \pi^{*}$ such that no perfect matching with minimum weight in $s\left(\pi^{*}\right)$ contains this edge. Assume the reverse, and let $\pi^{i}$ for $i=1, \cdots, \frac{n}{2}$ be a perfect matching with minimum weight $w\left(\pi^{*}\right)-r$ which contains edge $e_{i}$ in scenario $s\left(\pi^{*}\right)$ (note that possibly some $\pi^{i}$ are identical). Then, in scenario $s\left(\pi^{*}\right)$ we have:

$$
\sum_{i=1}^{\frac{n}{2}} w\left(\pi^{i} \backslash e_{i}\right)=\frac{n-2}{2} w\left(\pi^{*}\right)-\frac{n}{2} r
$$

On the other hand, the graph $G^{\prime}$ induced by $\cup_{i=1}^{\frac{n}{2}}\left(\pi^{i} \backslash e_{i}\right)$ is $\left(\frac{n}{2}-1\right)$-regular $\left(G^{\prime}\right.$ is considered as a multigraph, that is if an edge $(x, y)$ appears $p$ times in $\cup_{i=1}^{\frac{n}{2}}\left(\pi^{i} \backslash e_{i}\right)$, then there are $p$ parallel edges between $x$ and $y$ in $G^{\prime}$ ). Since $G^{\prime}$ is bipartite and $\left(\frac{n}{2}-1\right)$-regular, $G^{\prime}$ can be decomposed into $\left(\frac{n}{2}-1\right)$ matchings $\pi^{i}$ for $i=1, \ldots, \frac{n}{2}-1$. These matchings $\pi^{i}$ are perfect in $G$ and if $\pi^{\prime}$ is a matching of minimum weight in scenario $s\left(\pi^{*}\right)$ among the matchings $\pi^{\prime i}$ for $i=1, \ldots, \frac{n}{2}-1$, then the value of $\pi^{\prime}$ satisfies:

$$
\frac{n-2}{2} w\left(\pi^{\prime}\right) \leq \sum_{i=1}^{\frac{n}{2}} w\left(\pi^{i} \backslash e_{i}\right)
$$

Using equality (3) and inequality (4) we obtain $w\left(\pi^{\prime}\right) \leq w\left(\pi^{*}\right)-\left(1+\frac{2}{n}\right) r$, which is impossible for $r>0$ since $w\left(\pi^{\prime}\right) \geq w\left(\pi^{*}\right)-r$.

By applying $k$ times this method, we build $m^{k}$ instances such that $\mathcal{I}$ has an optimum regret at most $k$ iff (at least) one of the final instances has optimum regret 0 . Since we supposed that $\forall e \in E, l_{e} \geq k$ for the initial instance, all the interval lower bounds in the final instances are non-negative.

Our method seems to be quite general and may be fruitfully applied to other problems, but however not to all of them. Indeed, the property leading to Claim (iii) is no more true for some problems such as RST or RPM (in arbitrary graphs), and for them the question whether they are simple (according to the definition of [16]) or not remains open.

\section{Upper bounded number of interval intersections}

As previously mentioned, RST and RSP are fixed parameter tractable (FPT) when the parameter is the number of non degenerate intervals (with a brute force algorithm). Minimum spanning trees have special properties that leads to another easy cost structure: when all intervals are disjoint $\left(I_{e} \cap I_{f}=\emptyset\right.$ for any edges $e$ 
and $f$ ), any minimum spanning tree under any scenario is an optimum solution for RST [1]. Indeed, Kruskal's algorithm leads then to the same tree, independently of the scenario. This tree is optimal, and its regret is 0 . Note that, on the other hand, even if all intervals are $[0,1], \mathrm{RST}$ is NP-hard $[1,4]$. Here, we show that considering as parameter the number of intervals that intersect at least one other interval, RST is FPT. Although using brute force, the optimality of the algorithm is not obvious.

Proposition $3 R S T$ can be solved in time $O\left(2^{k} m \log m\right)$, where $k$ is the number of intervals that intersect at least one other interval.

Proof. Let $\mathcal{I}=\left(G, I_{E}\right)$ be an instance of RST where $G=(V, E)$ and $I_{e}=\left[l_{e}, u_{e}\right]$ for any $e \in E$. We define $J=\left\{I_{e_{1}}: \exists e_{2} \neq e_{1}, I_{e_{1}} \cap I_{e_{2}} \neq \emptyset\right\}$, and we set $k=|J|$. Let $J^{\prime} \subseteq J$. We want to compute the best (in terms of regret) spanning tree $\pi$ such that $\pi \cap E_{J}=E_{J^{\prime}}$ (where $E_{J}$ denotes the set of edges corresponding to intervals in $J)$. If $E_{J^{\prime}}$ contains a cycle, there is no such tree. If not, we proceed as follows: we remove from $E$ the set $E_{J \backslash J^{\prime}}$ and, considering $E_{J^{\prime}}$ as part of the spanning tree, we complete it by applying Kruskal's algorithm to the remaining graph (choosing any valuation $w(e) \in\left[l_{e}, u_{e}\right]$ since the output does not depend on the value of an edge $e \notin J)$. Let $\pi_{J^{\prime}}$ be the obtained solution.

Now, let $\pi$ be a spanning tree such that $\pi \cap E_{J}=E_{J^{\prime}}$. We want to prove that $R\left(\pi_{J^{\prime}}\right) \leq R(\pi)$. First, note that $\pi_{J^{\prime}}$ and $\pi$ agree on $E_{J}$. Then, under any scenario where $w(e)=u_{e}$ for $e \in E_{J^{\prime}}$ and $w(e)=l_{e}$ for $e \in E_{J \backslash J^{\prime}}$, Kruskal's algorithm will produce the same optimum solution $\pi^{*}$. In particular $\pi^{*}$ is optimal both in $s(\pi)$ and $s\left(\pi_{J^{\prime}}\right)$. However, $\pi^{*}$ has not the same value in these two scenarios. Then:

$$
R\left(\pi_{J^{\prime}}\right)-R(\pi)=w_{s\left(\pi_{J^{\prime}}\right)}\left(\pi_{J^{\prime}}\right)-w_{s\left(\pi_{J^{\prime}}\right)}\left(\pi^{*}\right)-\left(w_{s(\pi)}(\pi)-w_{s(\pi)}\left(\pi^{*}\right)\right)
$$

We upper bound this by considering each edge of the graph. If $\pi_{J^{\prime}}$ and $\pi$ agree on an edge $e$ (either take it or not), then the difference is 0 for this edge, since this edge has the same value in $s(\pi)$ and $s\left(\pi_{J^{\prime}}\right)$, and since we refer to the same tree $\pi^{*}$. Note that this includes all edges in $E_{J}$. If $\pi_{J^{\prime}}$ and $\pi$ disagree on $e$ :

- either $e$ is in $\pi_{J^{\prime}} \backslash \pi$. If $e$ is not in $\pi^{*}$, then in the regret it counts $u_{e}$ for $\pi_{J^{\prime}}$ $\left(u_{e}\right.$ for $\pi_{J^{\prime}}$ and 0 for $\pi^{*}$ ) and 0 for $\pi\left(0\right.$ for $\pi$ and 0 for $\left.\pi^{*}\right)$. If $e$ is in $\pi^{*}$, it counts 0 for $\pi_{J^{\prime}}$ and $-l_{e}$ for $\pi$. The loss (in terms of regret) from $\pi_{J^{\prime}}$ with respect to $\pi$ is therefore at most $u_{e}$;

- or $e$ is in $\pi \backslash \pi_{J^{\prime}}$. If $e$ is not in $\pi^{*}$, then it counts 0 for $\pi_{J^{\prime}}$ and $u_{e}$ for $\pi$. If $e$ is in $\pi^{*}$, it counts $-l_{e}$ for $\pi_{J^{\prime}}$ and 0 for $\pi$. Then, with respect to $\pi, \pi_{J^{\prime}}$ "wins" at least $l_{e}$.

Summing up these inequalities for all edges leads to:

$$
R\left(\pi_{J^{\prime}}\right)-R(\pi) \leq \sum_{e \in \pi_{J^{\prime}} \backslash \pi} u_{e}-\sum_{e \in \pi \backslash \pi_{J^{\prime}}} l_{e}
$$


Now, recall that $\pi$ and $\pi_{J^{\prime}}$ agree on $J$, and that the intervals not in $J$ do not intersect. Hence, whatever the value of edges not in $J, \pi_{J^{\prime}}$ will have a better value than $\pi$. This is true in particular when the weight of each $e \notin J$ is fixed to $u_{e}$ if $e$ is in $\pi_{J^{\prime}}$ and to $l_{e}$ otherwise. This means that

$$
\sum_{e \in \pi_{J^{\prime}} \backslash \pi} u_{e} \leq \sum_{e \in \pi \backslash \pi_{J^{\prime}}} l_{e}
$$

Equations (5) and (6) lead to the result that $\pi_{J^{\prime}}$ is the best tree $\pi$ such that $\pi \cap J=J^{\prime}$.

To conclude, we only have to consider each possible $J^{\prime} \subseteq J$, and take the best solution so computed. The global complexity is hence $2^{k} O(m \log m)$.

Note that for RSP, making assumptions on interval intersections does not simplify the problem.

Proposition $4 R S P$ is NP-hard even if there are no intersections between intervals.

\section{Upper bounded reduction complexity}

We now consider a particular class of directed acyclic graphs (DAGs), namely seriesparallel graphs. This class can be defined using the following kinds of reductions in a DAG: (1) a series reduction at $v$ is possible when $e_{1}=(u, v)$ is the unique edge into $v$ and $e_{2}=(v, w)$ is the unique edge out of $v$ : then $e_{1}$ and $e_{2}$ are replaced by $e=(u, w) ;(2)$ a parallel reduction at $u, w$ replaces two edges $e_{1}, e_{2}$ joining $u$ to $w$ by a single edge $e=(u, w)$. Two nodes $s$ and $t$ are distinguished as the source and the sink (st-DAG). A graph is said to be edge series-parallel (ESP) if it can be reduced to a single edge $(s, t)$ by using such reductions. Kasperski and Zielinski have recently shown that RSP is NP-hard in ESP graphs, but admits a pseudopolynomial algorithm in this case [14]. In this section, we extend this result to graphs close to be ESP. For the convenience of the reader, we first describe the basic principles of the pseudopolynomial algorithm for ESP graphs. It operates by applying a sequence of series and parallel reductions from the input graph $G=(V, E)$ to a single edge $(s, t)$. This sequence is given by an algorithm in $O(m)$ to recognize ESP graphs [17], where $m=|E|$. In a reduced graph, a subset $E_{i} \subseteq E$ is associated with every edge $e_{i}$. These subsets are defined recursively: the set $\{e\}$ is associated with every $e \in E$; let $e_{1}, e_{2}$ denote the edges involved in a reduction, then the set $E_{1} \cup E_{2}$ is associated with the new edge. For every edge $e_{i}$, the subgraph of $G$ induced by $E_{i}$ is denoted $G_{e_{i}}$. Let $u_{\pi}$ and $R(\pi)$ denote respectively the worst value and the max regret of a path $\pi$ in an induced subgraph $G_{e}$. The principle of the algorithm is, for each reduction yielding a new edge $e=(v, w)$, to keep only a minimal subset $P_{e}$ of nondominated paths from $v$ to $w$, where $\pi$ dominates $\sigma$ if $u_{\pi} \leq u_{\sigma}$ and $R(\pi) \leq R(\sigma)$ with at least a strict inequality. Indeed, those paths are potential subpaths of a $\operatorname{minmax}$ regret path from $s$ to $t$ in $G$. Initially, $P_{e}=\{e\}$ for every edge $e$. Then, 
for any new edge $e$ obtained by a reduction involving $e_{1}$ and $e_{2}$, set $P_{e}$ is computed from $P_{e_{1}} \cup P_{e_{2}}$ in a parallel reduction, and from $P_{e_{1}} \times P_{e_{2}}$ (concatenated paths) in a series reduction. When the sequence of reductions terminates, there is only a single edge $(s, t)$, and path $\pi^{*}=\arg \min _{\pi \in P_{(s, t)}} R(\pi)$ is a minmax regret path from $s$ to $t$ in $G$. Noticing that $\left|P_{e}\right|$ is upper bounded by $L_{\max }$, where $L_{\max }$ is the value of the longest path from $s$ to $t$ in $G$ over all scenarios, the authors, thanks to a recursive computation of $u$ and $R$ (avoiding shortest path computations from scratch when computing $R(\pi)$ for $\pi \in P_{e_{1}} \cup P_{e_{2}}$ or $\left.P_{e_{1}} \times P_{e_{2}}\right)$, establish that the running time is $O\left(m L_{\max }^{2}\right)$, and therefore pseudopolynomial.

We now extend this result to graphs close to be ESP. We first need to measure how far a graph is from being ESP. For that purpose, the notion of reduction complexity has been introduced [5]. It uses a third kind of reduction, called node reduction. Such a reduction can be performed at a node $v$ when $v$ has in-degree or out-degree 1: suppose $v$ has out-degree 1 , let $e_{1}=\left(u_{1}, v\right), \ldots, e_{\delta}=\left(u_{\delta}, v\right)$ be the edges into $v$ and $e_{\delta+1}=(v, w)$ be the edge out of $v$, then $\left\{e_{1}, \ldots, e_{\delta+1}\right\}$ is replaced by $\left\{e_{1}^{\prime}, \ldots, e_{\delta}^{\prime}\right\}$, where $e_{i}^{\prime}=\left(u_{i}, w\right)$ (the case where $v$ has in-degree 1 is symmetric). Note that every st-DAG can be reduced to a single edge $(s, t)$ by iterating the three types of reductions. The reduction complexity of a graph $G$ is defined as the minimum number of node reductions sufficient -along with series and parallel reductions- to reduce $G$ to $(s, t)$. There exists an $O\left(n^{2.5}\right)$ algorithm to compute an optimal reduction sequence [5] (i.e., involving a minimum number of node reductions), and hence to determine reduction complexity. Thanks to this, the result of Kasperski and Zielinski [14] can be extended:

Proposition $5 R S P$ can be solved in time $O\left(2^{k} m^{2} L_{\max }^{2}\right)$ in st-DAGs of reduction complexity $k$.

\section{Upper bounded treewidth and max degree}

The treewidth of a graph can be seen as a measure of how far it is from being a tree (the treewidth of a tree is 1). It is well-known that the treewidth of an (undirected) ESP graph is at most 2. A natural extension of the previous result is therefore to investigate the complexity of RSP in graphs of bounded treewidth (more precisely, in graphs whose corresponding undirected simple graph has a bounded treewidth). Clearly, RSP is polynomially solvable in a graph $G$ the treewidth of which is $k=1$ ( $G$ is a tree), or the max degree of which is $\Delta \leq 2$ ( $G$ is a set of cycles and/or chains). However, it is NP-hard when $k=2$ and $\Delta=3$ (since there is a polynomial reduction from the partition problem involving an ESP graph -without multiedgesof max degree 3 [14]). We show here its pseudopolynomiality for bounded $k$ and $\Delta$.

Proposition $6 R S P$ can be solved in time $O\left((n+m) 2^{\Delta(k+1)}\left((n-1) u_{\max }\right)^{k+1}\right)$ in graphs of treewidth $k$ and max degree $\Delta$, where $u_{\max }=\max _{(i, j) \in A} u_{i j}$.

Proof. Let $G=(V, A)$ denote a directed graph with a source node $s$ and a sink node $t$, and let $G^{\prime}=(V, E)$ denote the simple undirected graph obtained from $G$ 
by removing orientation of edges and by simplifying multiedges. Solving RSP in $G$ amounts to solve the following integer linear program (ILP) [11]:

$$
\begin{aligned}
& \min \sum_{(i, j) \in A} u_{i j} y_{i j}-x_{t} \\
& \text { s.t. } x_{j} \leq x_{i}+l_{i j}+\left(u_{i j}-l_{i j}\right) y_{i j} \quad \forall(i, j) \in A, \\
& \sum_{(j, k) \in A} y_{j k}-\sum_{(i, j) \in A} y_{i j}=\left\{\begin{array}{cl}
1 & \text { if } j=s \\
-1 & \text { if } j=t \\
0 & \text { if not }
\end{array} \quad \forall j \in V,\right. \\
& x_{s}=0, y_{i j} \in\{0,1\} \forall(i, j) \in A, x_{j} \in \mathbb{N} \forall j \in V .
\end{aligned}
$$

The interaction graph of an ILP includes a vertex for each variable of the program and an edge between two vertices if both corresponding variables appear in the same constraint. We now show that the program is solvable in pseudopolynomial time by applying a dynamic programming technique on a tree decomposition of the interaction graph $I G=(I, U)$, i.e. a labeled tree $(T, L)$ such that $(a)$ every node $t$ of $T$ is labeled by a non-empty subset $L(t)$ of $V$ s.t. $\cup_{t \in T} L(t)=V,(b)$ for every edge $\{i, j\} \in U$ there is a node $t$ of $T$ whose label $L(t)$ contains both $i$ and $j,(c)$ for every vertex $i \in I$ the nodes of $T$ whose labels include $i$ form a connected subtree of $T$. The width of a tree decomposition is $\max _{t \in T}|L(t)|-1$. The treewidth of $I G$ is the smallest $k$ for which $I G$ has a tree decomposition of width $k$. If the treewidth of a graph is bounded by a constant $k$, then a tree decomposition of treewidth at most $k$ can be constructed in linear time (in the number of nodes) [7]. This tree decomposition can itself be converted in linear time in a nice tree decomposition of the same width, i.e. a rooted tree decomposition such that each node has at most two children, with four types of nodes $t$ : leaf nodes with $|L(t)|=1$, join nodes with two children $t^{\prime}, t^{\prime \prime}$ s.t. $L(t)=L\left(t^{\prime}\right)=L\left(t^{\prime \prime}\right)$, introduce nodes with one child $t^{\prime}$ s.t. $L\left(t^{\prime}\right)=L(t) \cup\{v\}$ for some $v \in V$, forget nodes with one child $t^{\prime}$ s.t. $L(t)=L\left(t^{\prime}\right)-\{v\}$ for some $v \in V$. The proof of pseudopolynomiality of the approach is in three steps: $(i)$ we show that if the max degree of $G$ and the treewidth of $G^{\prime}$ are bounded by some constant, then the treewidth of $I G$ is bounded by some constant; (ii) we show how to solve by dynamic programming an ILP whose IG has a bounded treewidth; (iii) we show that the previous approach is pseudopolynomial since variables $x_{j}$ are upper bounded by $(n-1) u_{\max }$, where $u_{\max }=\max _{(i, j) \in A} u_{i j}$. Proof of $(i)$. Assume that $G^{\prime}$ has treewidth $k$ and $G$ has max degree $\Delta$. Note that $I G$ restricted to constraints (9) is the line graph of $G$, i.e., the graph where each vertex represents an edge of $G$ and any two vertices are adjacent iff their corresponding edges are incident. It can be shown that the treewidth of the line graph is at most $\Delta(k+1)-1[2]$. Assuming $(T, L)$ is a tree decomposition of width $k$ of $G^{\prime}$, the idea is to consider the labeled tree $\left(T, L^{\prime}\right)$ where $L^{\prime}(t)$ is the set of edges of $G$ incident to some node in $L(t)$. Indeed, one can show that $\left(T, L^{\prime}\right)$ is then a tree decomposition of the line graph [2]. We now show that $\left(T, L \cup L^{\prime}\right)$ is a tree decomposition of $I G$ (where we identify a vertex or an edge of $G$ with the corresponding variable in the ILP). For this purpose, one can consider the following 
partitions of $I$ and $U: I=X \cup Y$, where $X=\left\{x_{j}: j \in V\right\}$ and $Y=\left\{y_{i j}:(i, j) \in\right.$ $A\}$, and $U=U_{X} \cup U_{Y} \cup U_{X Y}$, where $U_{X}=\left\{\left[x_{i}, x_{j}\right]:(i, j) \in A\right\}, U_{Y}=\left\{\left[y_{j k}, y_{i j}\right]:\right.$ $(i, j) \in A,(j, k) \in A\}$ and $U_{X Y}=\left\{\left[x_{i}, y_{i j}\right],\left[x_{j}, y_{i j}\right]:(i, j) \in A\right\}$. Condition $(a)$ holds since $\cup_{t \in T} L(t)=X$ and $\cup_{t \in T} L^{\prime}(t)=Y$. Conditions $(b)$ and $(c)$ hold for edges of $U_{X}$ and for vertices in $X$ since $(T, L)$ is a tree decomposition of $G^{\prime}$. They also hold for edges of $U_{Y}$ and for vertices in $Y$ since $\left(T, L^{\prime}\right)$ is a tree decomposition of the line graph. Besides, condition (b) holds for edges of $U_{X Y}$ by construction of $L^{\prime}$. Hence, $\left(T, L \cup L^{\prime}\right)$ is a tree decomposition of $I G$. Furthermore, the treewidth of $I G$ is upper bounded by $\max _{t \in T} L(t)+\max _{t \in T} L^{\prime}(t)-1=k+\Delta(k+1)$.

Proof of $(i i)$. By using a method related to non-serial dynamic programming [6], we now show how to solve an ILP in the following general form:

$$
(P)\left\{\begin{array}{l}
\min \sum_{j=1}^{n} c_{j} x_{j} \\
\sum_{j=1}^{n} a_{i j} x_{j} \mathcal{R}_{i} b_{i} \text { where } \mathcal{R}_{i} \in\{\leq,=, \geq\} \quad \forall i \leq m \\
x_{j} \in D_{j} \quad \forall j \leq n
\end{array}\right.
$$

For this purpose, let us introduce the notion of subprogram of an ILP. For each node $t$ of $T, P(t)$ denotes the subprogram of $P$ restricted to the variables whose indices belong to $D(t)=\bigcup_{t^{\prime}} L\left(t^{\prime}\right)$ for $t^{\prime}=t$ or $t^{\prime}$ a descendant of $t$ :

$$
(P(t))\left\{\begin{array}{l}
\min \sum_{j \in D(t)} c_{j} x_{j} \\
\sum_{j=1}^{n} a_{i j} x_{j} \mathcal{R}_{i} b_{i} \quad \forall i:\left[\forall j,\left(a_{i j} \neq 0 \Rightarrow j \in D(t)\right)\right] \\
x_{j} \in D_{j}, \forall j \in D(t)
\end{array} \forall j\right.
$$

Given $t \in T$ and $\sigma: L(t) \rightarrow \Pi_{j \in L(t)} D_{j}$ an assignment of values to variables of $L(t)$, we denote by $R_{t}(\sigma)$ the minimum value of a feasible solution $x$ of $P(t)$ under the constraint $x_{j}=\sigma(j) \forall j \in L(t)$. One sets $R_{t}(\sigma)=+\infty$ if no feasible solution of $P(t)$ is compatible with $\sigma$. The dynamic programming algorithm consists of traversing the nice tree decomposition in a bottom up manner, and computing recursively the tables $R_{t}$ for each $t \in T$, where table $R_{t}$ has an entry $R_{t}(\sigma)$ for each possible assignment $\sigma$ : let $t$ be a leaf node, say $L(t)=\{j\}$, then $R_{t}(\sigma)=c_{j} \sigma(j)$; let $t$ be a join node with two children $t^{\prime}$ and $t^{\prime \prime}$, then $R_{t}(\sigma)=R_{t^{\prime}}(\sigma)+R_{t^{\prime \prime}}(\sigma)-\sum_{j \in L(t)} c_{j} \sigma(j)$; let $t$ be an introduce node, say $L(t)=L\left(t^{\prime}\right) \cup\{j\}$, then $R_{t}(\sigma)=+\infty$ if $\sigma$ violates a constraint of $P(t)$, otherwise $R_{t}(\sigma)=R_{t^{\prime}}\left(\sigma_{t^{\prime}}\right)+c_{j} \sigma(j)$ where $\sigma_{t^{\prime}}$ denotes assignment $\sigma$ restricted to the variables in $L\left(t^{\prime}\right)$; let $t$ be a forget node, say $L(t)=L\left(t^{\prime}\right)-\{j\}$, then $R_{t}(\sigma)=\min _{d_{j} \in D_{j}}\left\{R_{t^{\prime}}\left(\sigma^{\prime}\right): \sigma^{\prime}(k)=\sigma(k) \forall k \neq j\right.$ and $\left.\sigma^{\prime}(j)=d_{j}\right\}$. The optimum is $\min _{\sigma} R_{r}(\sigma)$ at the root node $r$ of the nice tree decomposition.

Proof of (iii). We have $|I|=n+m$ since there are $n x_{i}$ 's and $m y_{i j}$ 's in the ILP formulation of RSP. There are therefore $O(n+m)$ nodes in the nice tree decomposition. Noticing that a table $R_{t}$ can be computed in time $O\left(2^{\Delta(k+1)}\left((n-1) u_{\max }\right)^{k+1}\right)$ since there are at most $\Delta(k+1)$ boolean variables and $k+1$ integer variables in $L(t)$, the result follows. 
This approach based on properties of the interaction graph of an ILP formulation is quite general, and can be also fruitfully applied to RBPM. As in Section 2, for any instance of RBPM, we assume that there exists a perfect matching.

Proposition 7 RBPM can be solved in time $O\left((n+m) 2^{\Delta(k+1)}\left((n+1) u_{\max }\right)^{k+1}\right)$ in graphs of treewidth $k$ and max degree $\Delta$, where $u_{\max }=\max _{(i, j) \in E} u_{i j}$.

\section{Concluding remarks}

Several results given in this paper deserve to our opinion further research. For instance, we conjecture that RSP, as well as other problems, can be pseudopolynomially solved in graphs with bounded treewidth (without any degree restriction). Alternatively, devising a general method for solving in polynomial time any problem with bounded minmax regret could be very appealing, but the existence of such a method seems quite hypothetical to us.

Besides, the issue we considered here can also be investigated in the discrete scenario model. In that model, each edge $e$ is valued by $\left(s_{1}^{e}, \cdots, s_{b}^{e}\right)$. For example, the robust shortest path and spanning tree problems can be trivially solved under the minmax criterion when the set of valuations is comonotone, i.e. $s_{i}^{e} \leq s_{j}^{e} \Rightarrow$ $s_{i}^{f} \leq s_{j}^{f}$ for any $i, j$ and $e, f$. Indeed, the value of every solution is maximized under the same scenario. Then, one can measure the distance from comonotony as the minimum number of edges the removal of which leads to a comonotone instance. Interestingly enough, it can be shown that, even if the distance from comonotony is 1 , and even if there are only 2 scenarios, the robust shortest path and minimum spanning tree problems are NP-hard.

\section{References}

[1] I. D. Aron and P. Van Hentenryck. On the complexity of the robust spanning tree problem with interval data. Operations Research Letters, 32:36-40, 2004.

[2] A. Atserias. On digraph coloring problems and treewidth duality. URL www.lsi.upc.es/〜atserias/, 2006.

[3] I. Averbakh. On the complexity of a class of combinatorial optimization problems with uncertainty. Mathematical Programming, Ser. A 90:263-272, 2001.

[4] I. Averbakh and V. Lebedev. Interval data minmax regret network optimization problems. Discrete Applied Mathematics, 138:289-301, 2004.

[5] W. W. Bein, J. Kamburowski, and M. F. M. Stallmann. Optimal reduction of two-terminal directed acyclic graphs. SIAM J. on Computing, 21(6):11121129, 1992.

[6] U. Bertele and F. Brioschi. Nonserial Dynamic Programming. Academic Press, 1972. 
[7] H. L. Bodlaender. A linear-time algorithm for finding tree-decompositions of small treewidth. SIAM J. on Computing, 25(6):1305-1317, 1996.

[8] R. G. Downey and M. R. Fellows. Parameterized Complexity. Springer, 1999.

[9] B. Escoffier, J. Monnot, and O. Spanjaard. Some tractable instances of interval data minmax regret problems: bounded distance from triviality. Technical Report 265, Cahiers de recherche, LAMSADE, 2007. URL http://www.lamsade.dauphine.fr/cahiers/PDF/cahierLamsade265.pdf.

[10] J. Guo, F. Hüffner, and R. Niedermeier. A structural view on parameterizing problems: Distance from triviality. In IWPEC 2004, volume 3162 of LNCS, pages $162-173,2004$.

[11] O. E. Karasan, M. C. Pinar, and H. Yaman. The robust shortest path problem with interval data. Technical report, Bilkent Univ., Dpt of Industrial Engineering, 2001.

[12] A. Kasperski and P. Zielinski. Minimizing maximal regret in the linear assignment problems with interval costs. Technical Report 007, Instytut Matematyki Wroclaw, 2004.

[13] A. Kasperski and P. Zielinski. An approximation algorithm for interval data minmax regret combinatorial optimization problems. Information Processing Letters, 97:177-180, 2006.

[14] A. Kasperski and P. Zielinski. The robust shortest path problem in seriesparallel multidigraphs with interval data. Operations Research Letters, 34:69$76,2006$.

[15] P. Kouvelis and G. Yu. Robust Discrete Optimization and Its Applications. Kluwer Academic Publishers, 1997.

[16] A. Paz and S. Moran. Non deterministic polynomial optimisation problems and their approximation. Theoretical Computer Science, 95:251-277, 1981.

[17] J. Valdes, R. Tarjan, and E. Lawler. The recognition of series-parallel digraphs. SIAM J. on Computing, 11(2):298-313, 1982. 